\title{
Trend analysis of land surface temperatures using time series segmentation algorithm
}

\author{
Nini Wang ${ }^{\mathrm{a}, \mathrm{b}}$, Jun Xia ${ }^{\mathrm{a}, \mathrm{c}, *}$, Jianchuan Yin ${ }^{\mathrm{d}}$ and Xiaodong Liu ${ }^{\mathrm{b}}$ \\ ${ }^{a}$ Key Laboratory of Water Cycle and Related Land Surface Processes, Institute of Geographic Sciences \\ and Natural Resources Research, Chinese Academy of Sciences, Beijing, China \\ ${ }^{\mathrm{b}}$ Department of Mathematics, Dalian Maritime University, Dalian, Liaoning, China \\ ${ }^{\mathrm{c}}$ State Key Laboratory of Water Resources and Hydropower Engineering Science, Wuhan University, \\ Wuhan, Hubei, China \\ ${ }^{\mathrm{d}}$ Navigation College, Dalian Maritime University, Dalian, Liaoning, China
}

\begin{abstract}
Detecting temporal and spatial trends of annual and seasonal land surface temperature (LST) can contribute to study the effect of climate change and climate variability on temperature behaviors both in time and space. The temporal and spatial gridded dataset of monthly mean LST series produced by Berkeley Earth were chosen for the analysis of LST trends. The dynamic programming (DP) based segmentation algorithm is a fast and efficient time series segmentation algorithm which can identify multiple change points in a given time series. Multiple change points of annual and seasonal LST average anomaly time series during the period 1880-2013 (reference to the 20th century average) were identified by the DP based time series segmentation algorithm. Schwarz's Bayesian information criterion (BIC) was applied to automatically determine the optimal segmentation order. BIC selected one change point for annual and seasonal average LST except for the autumn season's. Regardless of the number of change points, at the first segment, trends are always increasing and at the last, they are sharply increasing except for the winter season. Moreover, all the change points locate around El Niño years, La Niña years, and phase transition years of the Pacific decadal oscillation (PDO). Based on optimal time series segmentation results selected by BIC, the spatial distributions of linear trends (slope estimates) of annual and seasonal LST anomalies corresponding to different homogeneous periods are showed. Comparing to adjacent segment, the recent warming trends not only appear in Greenland and the surrounding area, but also dominate most parts of land surface, and significant warming trends appear in high latitude regions of the Northern Hemisphere.
\end{abstract}

Keywords: Time series segmentation, temporal-spatial variability, trend analysis, climate change

\section{Introduction}

As a basic climatic factor, land surface temperature (LST) is a vital factor influencing other meteorological variables such as runoff, precipitation and pressure $[1,2]$. Nowadays, global warming aggravates the depletion of the world's available water resources $[3,4]$.

Climate change may cause the shift of the statistical characteristics (mean, median and variance etc.) of

\footnotetext{
${ }^{*}$ Corresponding author. Jun Xia. E-mail: xiaj@igsnrr.ac.cn.
}

LST [5-8]. LST is sensitive to climate change, and its trend and jump changes are temporally and spatially non-uniform [9-11]. In order to better understand climate change, a number of studies have examined LST change and variability on global and regional scales [3, 7, 12-19].

However, high-resolution gridded LST data sets are often limited in length. In order to further understand LST variability, Berkeley Earth has constructed longer gridded LST data. Berkeley Earth Surface Temperature (BEST) data incorporates more land stations than other available Earth's surface temperature 
data sets, such as USA's NOAA and NASA, and UK's CRU and Hadley Centre. Due to its spatially and temporally high resolution, BEST is an ideal data to assess the variability of global LST.

Trend analysis becomes the great importance method to detect LST. The most common approach to estimate trend of a given LST time series is simple linear regression, which fits time series by a straight line. This method can only find an overall linear trend, and can not identify the multiple change points between consecutive periods with different trends $[3,20]$. To address this problem, data mining methods for finding the evolution of LST on different temporal and spatial scales are necessary [21-29].

Time series segmentation is used to divide a given time series into homogeneous subseries with different statistical characteristics so that the mean values of two consecutive subseries are heterogeneous. A number of studies about time series segmentation algorithm have been proposed to analyze variability in hydro-meteorological time series [9, 10, 30-37].

Hubert proposed an automatic offline segmentation procedure to split a given time series into multiple segments [36]. The sum of the squared deviation of the within-segment data from their respective segment's average was defined as the segmentation cost which is used as the criterion to decide whether a change point exists $[9,10]$. Based on the concept of [36], Aksoy et al. presented a branchand-bound based algorithm (denoted as AUG), which can efficiently segment long time series without the blocking and pruning procedure [34, 35]. Based on the same concept of [36], Kehagias et al. proposed a dynamic programming based segmentation algorithm (denoted as DP), which is a non-parametric global optimal algorithm and can detect shift trends without knowing the number of segmentation order and the positions of change points [33].

This study aims to detect temporal and spatial trends of annual and seasonal LST on Berkeley Earth LST data. The DP based time series segmentation algorithm was applied to determine multiple change points of annual and seasonal average LST anormaly time series. Then Schwarz's Bayesian information criterion (BIC) was applied to automatically determine the optimal segmentation order. Based on the optimal segmentation results selected by BIC, the spatial distributions of linear trends (slope estimates) of annual and seasonal LST anomalies corresponding to different homogeneous periods were displayed.

The study is arranged as follows. Section 2 describes data sets together with the DP based time series segmentation algorithm. Section 3 presents the temporal-spatial trend analysis of annual and seasonal LST anomaly series. Conclusions are finally drawn in Section 4.

\section{Data and methods}

Berkeley Earth has reanalyzed the Earth's surface temperature record, and obtained a long and accurate monthly mean LST record back to 1701 . They released their analysis, programs and established open-access database with all the LST time series data and gridded data used in the BEST study. All the Berkeley temperatures are in Celsius and reported as anomalies relative to the Jan. 1951-Dec. 1980 average.

Annual and monthly average temperatures is over 1750-2015 period with the $95 \%$ confidence interval for statistical and spatial under-sampling effects. Monthly gridded temperatures are at a $1^{\circ} \times 1^{\circ}$ (latitude $\times$ longitude) resolution over 1880-2015 period without confidence interval. For more information on the BEST study, see http://www.berkeleyearth.org.

In this paper, annual and seasonal land surface temperature anomalies for the period Jan. 1880-Feb. 2014 are given compared to their 20th century average, which counts for 134 years. The study area is chosen from $60^{\circ} \mathrm{S}-90^{\circ} \mathrm{N}$ to $180^{\circ} \mathrm{W}-180^{\circ} \mathrm{E}$. Antarctica were not considered because of the low quality of data. The seasonal temperatures were generated by the arithmetic means of three consecutive months that belong to four climatological seasons: spring (from March to May), summer (from June to August), autumn (from September to November), and winter (from December of this year to February of the next year).

The annual and seasonal average LST anomalies were analyzed with the DP based segmentation algorithm. Details about the DP algorithm can be found in many publications [10, 32-36].

A $K$-th order segmentation of a given time series $\mathbf{x}=\left(x_{1}, x_{2}, \ldots, x_{N}\right)$ is a sequence $\mathbf{t}=\left(t_{0}, t_{1}\right.$, $\left.\ldots, t_{K}\right)$ which satisfies $0=t_{0}<t_{1}<\cdots<t_{K-1}<$ $t_{K}=N$. The set of all segmentations of $\mathbf{x}$ is denoted by $\mathrm{T}$ and the set of all segmentations of order $K$ by $\mathrm{T}_{K} . \mathrm{T}=\bigcup_{K=1}^{N} \mathrm{~T}_{K}$. The times $t_{0}, t_{1}, \ldots, t_{K}$ are called change points and $K$ is called the segmentation order.

Time series segmentation is represented as an optimization problem. The segmentation cost functin $J(\mathbf{t})$ is defined as: 


$$
J(\mathbf{t})=\sum_{k=1}^{K} d_{t_{k-1}+1, t_{k}},
$$

where $d_{s, t}($ for $0 \leq s<t \leq N)$ is the segment error corresponding to segment $[s, t]$. The segment error $d_{s, t}$ depends on the data $x_{1}, x_{2}, \ldots, x_{N}$, which is defined as:

$$
d_{s, t}=\sum_{\tau=s}^{t}\left(x_{\tau}-\hat{x}_{\tau}\right)^{2} .
$$

where the optimal $\hat{x}_{\tau}$ is given by $\hat{x}_{\tau}=\sum_{\tau=s}^{t} x_{\tau} /(t-$ $s+1)$, i.e. the empirical mean. For constant regression:

$$
x_{\tau}=a_{1}+e_{t},
$$

where $a_{1}$ is regression coefficient, and $e_{t}$ is stochastic error. For linear regression:

$$
x_{\tau}=a_{1}+a_{2} \cdot t+e_{t},
$$

where $a_{1}, a_{2}$ are regression coefficient, and $e_{t}$ is stochastic error.

The optimal segmentation $\hat{\mathbf{t}}=\left(\hat{t}_{0}, \hat{t}_{1}, \ldots, \hat{t}_{K}\right)$ is defined as

$$
\hat{\mathbf{t}}=\arg \min _{\mathbf{t} \in \mathrm{T}} J(\mathbf{t})
$$

and the $K$ th order optimal segmentation order $\hat{\mathbf{t}}^{(K)}=$ $\left.\left.\left(\hat{t}_{0}^{(K)}\right), \hat{t}_{1}^{(K)}\right), \ldots, \hat{t}_{K}^{(K)}\right)$ is defined as

$$
\hat{\mathbf{t}}^{(K)}=\arg \min _{\mathbf{t} \in \mathrm{T}_{K}} J(\mathbf{t}) .
$$

The corresponding minimum segmentation cost is denoted by $\hat{J}_{K}=J\left(\hat{\mathbf{t}}^{K)}\right)$. We have

$$
\hat{J}_{1} \geq \hat{J}_{2} \geq \cdots \geq \hat{J}_{N}=0 .
$$

Note that $\hat{J}_{N}=0$ means every segment only contains a single data. But this segmentation result does not convey any information of the time series. This is a segmentation order selection problem, which is a exceptional case of model order selection problem.

Noted that the segmentation order increases exponentially with the number of time series $N$, Kehagias et al. presented the DP based time segmentation algorithm [33]. The DP based segmentation algorithm efficiently calculates the optimal $k$ th-order segmentation for $k=1,2, \ldots, K$ as follows: Consider an optimal $K$ th-order segmentation of a given time series $x_{1}, x_{2}, \ldots, x_{t}$ and suppose the last segment is $[s+1, t]$. The first $K-1$ segments form the optimal segmentation of time series $x_{1}, x_{2}, \ldots, x_{s}$. Specifically, if $c_{t}^{(k)}$ is the minimum segmentation cost of $k$ th order segmentation of time series $x_{1}, x_{2}, \ldots, x_{t}$, then

$$
c_{t}^{(k)}=c_{s}^{(k-1)}+d_{s+1, t} .
$$

According to Equation (8), the optimal segmentation costs and the corresponding optimal change points for $k=1,2, \ldots, K$ can be calculated by the typical DP approach. On termination, the optimal segmentation cost for the $K$ th-order segmentation has been computed as

$$
c_{T}^{K}=J\left(\hat{\mathbf{t}}_{K}\right)=\min _{\mathbf{t} \in \mathbf{T}_{K}} J(\mathbf{t}) .
$$

Then, by backtracking, the optimal segmentation $\hat{\mathbf{t}}_{k}=\left(\hat{t}_{1}, \ldots, \hat{t}_{k}\right), k=1,2, \ldots, K$ have been computed recursively.

A quick procedure for computing the costs $d_{s, t}$ is the following recursive formulation

$$
\begin{aligned}
d_{s, t+1}= & d_{s, t}+(t-s+1)\left(\mu_{s, t}-\mu_{s, t+1}\right)^{2} \\
& +\left(x_{t+1}-\mu_{s, t+1}\right)^{2},
\end{aligned}
$$

where

$$
\mu_{s, t+1}=\frac{(t-s+1) \mu_{s, t}+x_{t+1}}{t-s+2}
$$

Determining the optimal segmentation order (the number of segments) is the next step in the segmentation procedure. In this paper, Schwarz's Bayesian information criterion (BIC) is applied to automatically determine the optimal number of segments. The $\mathrm{BIC}$ criterion is defined as

$$
\tilde{J}(K)=N \log \left(\frac{\hat{J}(K)}{N-1}\right)+R_{K} \log (N),
$$

where $R_{K}$ is the number of parameters of the optimal $K$-th order segmentation model and the optimal segmentation order is the value of $K$ which minimizes $\tilde{J}(K)$.

\section{Results}

In this section, annual and seasonal LST anomalies from Jan. 1880 to Feb. 2014 (deviations from the 20th century average) are analyzed by the DP based time series segmentation algorithm to quantify temporal and spatial LST trend changes. The main results contain two aspects. The first aspect is to analyze the trend changes of average LST anomalies at different time scales (i.e. annual and seasonal scales). The second one is to analyze the spatial trends of annual and 
seasonal LST anomalies based on the temporal trend change results.

\subsection{Annual and seasonal average LST variations from Jan. 1880 to Feb. 2014}

Figure 1 illustrates the temporal evolution of annual and seasonal average LST anomalies during Jan. 1880 to Feb. 2014 (reference period 1901-2000). Negative anomalies and positive anomalies separately represent the cooler and the warmer period relative to the basic period 1901-2000. As can be seen, all temperature anomalies prior to 1920s-1930s are lower than their 20th century average. After 1970s-1980s, all temperature anomalies are higher than their 20th century average.

The statistical characteristics of mean, range, standard deviation (SD), coefficient of variation (CV), skewness, and kurtosis of annual and seasonal average LST anomaly time series from Jan. 1880 to Feb. 2014 (reference period 1901-2000) are given in Table 1.

Figure 2 shows the histograms of annual and seasonal average LST anomaly time series. As can be
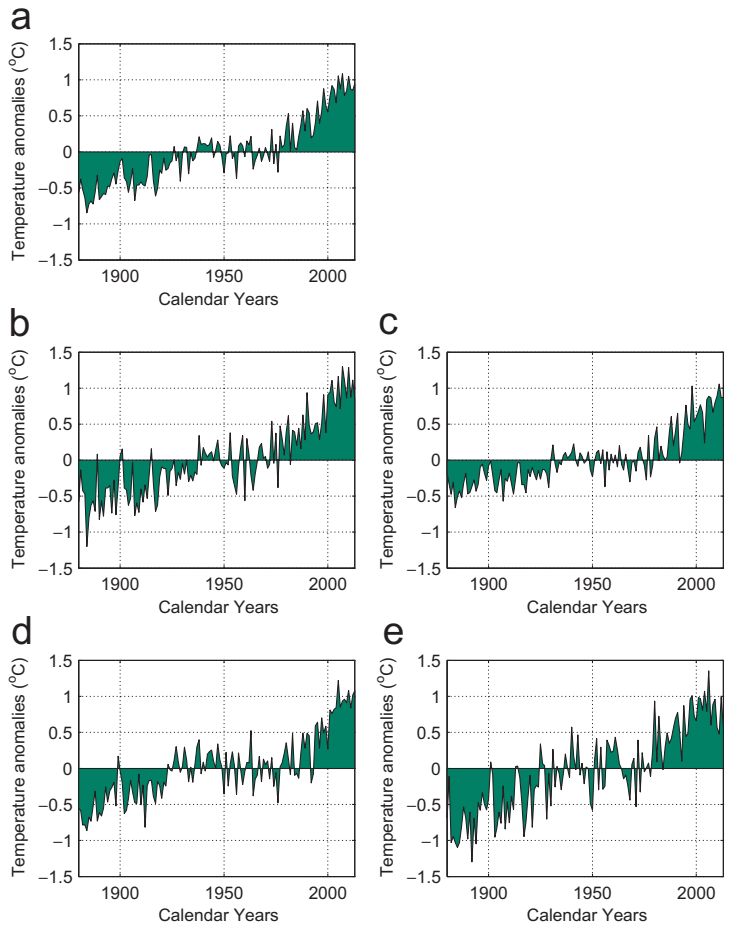

Fig. 1. Annual and seasonal average LST anomaly time series during Jan. 1880 to Feb. 2014 (reference period 1901-2000) (a-Annual, b-Spring, c-Summer, d-Autumn and e-Winter).
Table 1

Statistical characteristics of annual and seasonal average LST anomaly time series

\begin{tabular}{|c|c|c|c|c|c|c|}
\hline Period & $\begin{array}{c}\text { Mean } \\
\left({ }^{\circ} \mathrm{C}\right)\end{array}$ & $\begin{array}{c}\text { Range } \\
\left({ }^{\circ} \mathrm{C}\right)\end{array}$ & $\begin{array}{l}\text { SD } \\
\left({ }^{\circ} \mathrm{C}\right)\end{array}$ & $\mathrm{CV}$ & $\begin{array}{c}\text { Skewn- } \\
\text { ess }\end{array}$ & Kurtosis \\
\hline Annual & 0.0064 & 1.9310 & 0.4431 & 69.3412 & 0.5362 & 2.7616 \\
\hline Spring & 0.0151 & 2.5007 & 0.5077 & 33.7320 & 0.4473 & 2.8288 \\
\hline Summer & 0.0240 & 1.7180 & 0.3753 & 15.6376 & 0.9064 & 3.2890 \\
\hline Autumn & 0.0143 & 2.0853 & 0.4497 & 31.5473 & 0.4787 & 2.9532 \\
\hline Winter & -0.0301 & 2.6503 & 0.5671 & 18.8222 & 0.0575 & 2.3891 \\
\hline
\end{tabular}

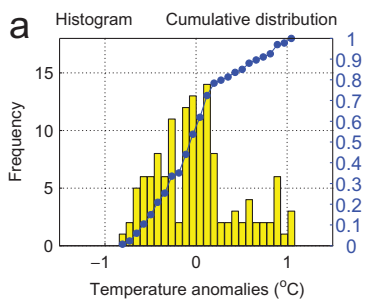

b Histogram Cumulative distribution
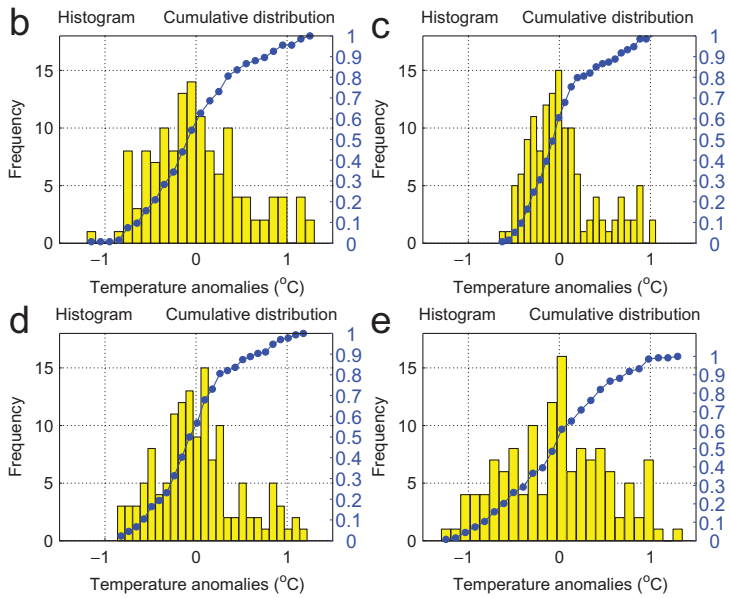

Fig. 2. Histogram and cumulative distribution for annual and seasonal average LST anomalies (reference period 1901-2000) (a-Annual, b-Spring, c-Summer, d-Autumn and e-Winter).

seen, all the records are not normally distributed because of their tails (positive skew).

Table 2 exhibits the change points of the optimal segmentations up to the 4th-order obtained by the DP based segmentation algorithm for annual and seasonal average LST anomaly time series. The overall linear trends show that the annual and seasonal average LST anomaly time series display increasing trend and the uptrend rates are $0.0101^{\circ} \mathrm{C} /$ year, $0.0109^{\circ} \mathrm{C} /$ year, $0.0079^{\circ} \mathrm{C} /$ year, $0.0092^{\circ} \mathrm{C} /$ year and $0.0122^{\circ} \mathrm{C} /$ year, respectively. The uptrend rate of winter temperature anomaly time series is higher than in autumn, spring and summer seasons.

Table 2 also shows the BIC values, which can determine the optimal segmentation order. The opti- 
Table 2

Change points in the optimal segmentations of annual and seasonal average LST anomaly time series for orders

$$
K=1,2,3,4
$$

\begin{tabular}{|c|c|c|c|c|c|c|c|}
\hline \multirow{2}{*}{$\frac{\text { Period }}{\text { Annual }}$} & \multirow{2}{*}{$\frac{K}{1}$} & \multicolumn{5}{|c|}{ Change points } & \multirow{2}{*}{$\frac{\text { BIC }}{-406.57}$} \\
\hline & & 1880 & 2013 & & & & \\
\hline & 2 & 1880 & 1963 & 2013 & & & -458.68 \\
\hline & 3 & 1880 & 1948 & 1963 & 2013 & & -455.78 \\
\hline & 4 & 1880 & 1902 & 1948 & 1963 & 2013 & -450.22 \\
\hline \multirow[t]{4}{*}{ Spring } & 1 & 1880 & 2013 & & & & -327.95 \\
\hline & 2 & 1880 & 1953 & 2013 & & & -352.27 \\
\hline & 3 & 1880 & 1954 & 1959 & 2013 & & -344.10 \\
\hline & 4 & 1880 & 1883 & 1889 & 1953 & 2013 & -338.04 \\
\hline \multirow[t]{4}{*}{ Summer } & 1 & 1880 & 2013 & & & & -396.01 \\
\hline & 2 & 1880 & 1966 & 2013 & & & -450.99 \\
\hline & 3 & 1880 & 1929 & 1979 & 2013 & & -447.86 \\
\hline & 4 & 1880 & 1929 & 1977 & 1981 & 2013 & -441.65 \\
\hline \multirow[t]{4}{*}{ Autumn } & 1 & 1880 & 2013 & & & & -336.07 \\
\hline & 2 & 1880 & 1963 & 2013 & & & -374.30 \\
\hline & 3 & 1880 & 1922 & 1973 & 2013 & & -383.05 \\
\hline & 4 & 1880 & 1901 & 1922 & 1973 & 2013 & -381.48 \\
\hline \multirow[t]{4}{*}{ Winter } & 1 & 1880 & 2013 & & & & -297.57 \\
\hline & 2 & 1880 & 1963 & 2013 & & & -301.53 \\
\hline & 3 & 1880 & 1963 & 2006 & 2013 & & -295.47 \\
\hline & 4 & 1880 & 1881 & 1963 & 2006 & 2013 & -288.43 \\
\hline
\end{tabular}

mal segmentations selected by the BIC for annual and seasonal average LST anomaly time series are listed by bold letters. The BIC selects the segmentation $(1880,1963,2013)$ for annual, the segmentation $(1880,1953,2013)$ for spring season, the segmentation $(1880,1966,2013)$ for summer season, the segmentation $(1880,1922,1973,2013)$ for autumn season, and the segmentation (1880/81, $1963 / 64,2013 / 14$ ) for winter season. The optimal segmentations selected by the BIC reveal that all the average LST anomaly time series except the autumn season's are divided into two segments. This behavior is unobserved when the traditional trend analysis method (only one segment) is applied.

Tables 3 and 4 separately show slope and intercept estimates of parital trends of annual and seasonal average LST anomaly series for orders $K=1,2,3,4$ obtained from the DP based segmentation algorithm. In both tables, the optimal segmentations selected by the BIC are listed by bold letters.

Figure 3 shows that annual average LST anomaly time series (deviations from the 20th century average), and change points identified by the DP based segmentation algorithm together with partial trend signs up to the 4th-order. It can be seen that LST on annual scale exhibits an increasing trend along the whole period (1880-2013). The 2th-order optimal segmentation shows that the change point in 1963. The 3th-order optimal segmentation shows that the first warming spell started in 1880 and ended in 1948,
Table 3

Slope estimates of linear trends in the optimal segmentations of annual and seasonal average temperature anomaly time series for orders $K=1,2,3,4$. (Bold letters indicate optimal segmentations selected by the BIC)

\begin{tabular}{lrrrrr}
\hline Period & $K$ & \multicolumn{5}{c}{ Partial linear trends (slopes) } \\
\hline Annual & 1 & 0.0101 & & & \\
& $\mathbf{2}$ & $\mathbf{0 . 0 0 9 3}$ & $\mathbf{0 . 0 2 4 4}$ & & \\
& 3 & 0.0113 & 0.0183 & 0.0244 & \\
Spring & 4 & 0.0190 & 0.0142 & 0.0183 & 0.0244 \\
& 1 & 0.0109 & & & \\
& $\mathbf{2}$ & $\mathbf{0 . 0 1 0 0}$ & $\mathbf{0 . 0 2 2 4}$ & & \\
& 3 & 0.0097 & 0.2024 & 0.0240 & \\
& 4 & 0.0017 & 0.1919 & 0.0110 & 0.0224 \\
Summer & 1 & 0.0079 & & & \\
& $\mathbf{2}$ & $\mathbf{0 . 0 0 5 6}$ & $\mathbf{0 . 0 2 4 0}$ & & \\
& 3 & 0.0040 & -0.0016 & 0.0247 & \\
& 4 & 0.0040 & -0.0010 & 0.2459 & 0.0279 \\
Autumn & 1 & 0.0092 & & & \\
& 2 & 0.0098 & 0.0259 & & \\
& $\mathbf{3}$ & $\mathbf{0 . 0 0 9 7}$ & $-\mathbf{0 . 0 0 1 9}$ & $\mathbf{0 . 0 3 2 3}$ & \\
Winter & 4 & 0.0318 & 0.0110 & -0.0019 & 0.0323 \\
& 1 & 0.0122 & & & \\
& $\mathbf{2}$ & $\mathbf{0 . 0 1 3 1}$ & $\mathbf{0 . 0 2 4 0}$ & & \\
& 3 & 0.0131 & 0.0301 & -0.0143 & \\
& 4 & -0.0010 & 0.0139 & 0.0301 & -0.0143 \\
\hline
\end{tabular}

Table 4

Intercept estimates of linear trends in the optimal segmentations of the average temperature anomaly time series, for orders $K=1,2,3,4$. (Bold letters indicate optimal segmentations selected by the BIC)

\begin{tabular}{lrrrrr}
\hline Period & $K$ & \multicolumn{5}{c}{ Partial linear trends (slopes) } \\
\hline Annual & 1 & -19.5987 & & & \\
& $\mathbf{2}$ & $\mathbf{- 1 8 . 0 5 6 6}$ & $\mathbf{- 4 8 . 2 0 9 3}$ & & \\
& 3 & -21.9593 & -35.7446 & -48.2093 & \\
& 4 & -36.4270 & -27.5387 & -35.7446 & -48.2093 \\
Spring & 1 & -21.2768 & & & \\
& $\mathbf{2}$ & $-\mathbf{1 9 . 4 7 6 8}$ & $-\mathbf{4 4 . 1 6 3 0}$ & & \\
& 3 & -18.8306 & -396.2213 & -47.3152 & \\
& 4 & -3.5800 & -362.7257 & -21.3213 & -44.1630 \\
Summer & 1 & -15.3360 & & & \\
& $\mathbf{2}$ & $-\mathbf{1 0 . 8 6 1 0}$ & $\mathbf{- 4 7 . 3 4 4 4}$ & & \\
& 3 & -7.8849 & 3.1501 & -48.8113 & \\
& 4 & -7.8849 & 2.0327 & -486.6433 & -55.2317 \\
Autumn & 1 & -17.9697 & & & \\
& 2 & -19.1045 & -51.0938 & & \\
& $\mathbf{3}$ & $-\mathbf{1 8 . 9 3 9 8}$ & $\mathbf{3 . 7 9 1 2}$ & $-\mathbf{6 4 . 0 1 2 2}$ & \\
& 4 & -60.5857 & -21.3266 & 3.7912 & -64.0122 \\
Winter & 1 & -23.7364 & & & \\
& $\mathbf{2}$ & $-\mathbf{2 5 . 5 2 1 7}$ & $-\mathbf{4 7 . 2 2 1 8}$ & & \\
& 3 & -25.5217 & -59.3725 & 29.4581 & \\
& 4 & 0.5740 & -27.0805 & -59.3725 & 29.4581 \\
\hline
\end{tabular}

the second warming spell started in 1849 and ended in 1963, while the third warming spell started in 1964 and ended in 2013. The 4th-order optimal segmentation shows that the change points in 1902, 1948 and 1963. In the recent years (1964-2013), the warming trend has accelerated at a rate of $0.0244^{\circ} \mathrm{C} /$ year. 


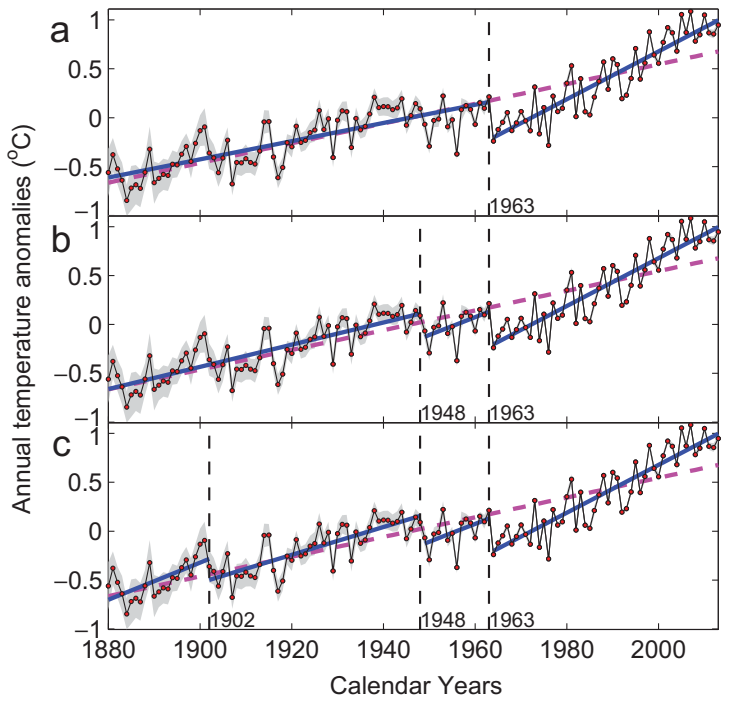

Fig. 3. Segmentation results of annual average LST anomaly time series during 1880-2013 (reference period 1901-2000) for 2thorder (top), 3th-order (middle), and 4th-order (down) together with partial trends. Grey shading is the uncertainties; Red dot lines denote temperature anomaly time series; Magenta dotted lines denote linear trends over the whole period; Blue solid lines denote partial linear trends obtained by the DP based segmentation algorithm; Vertical blank dotted lines provide the locations of the change points.

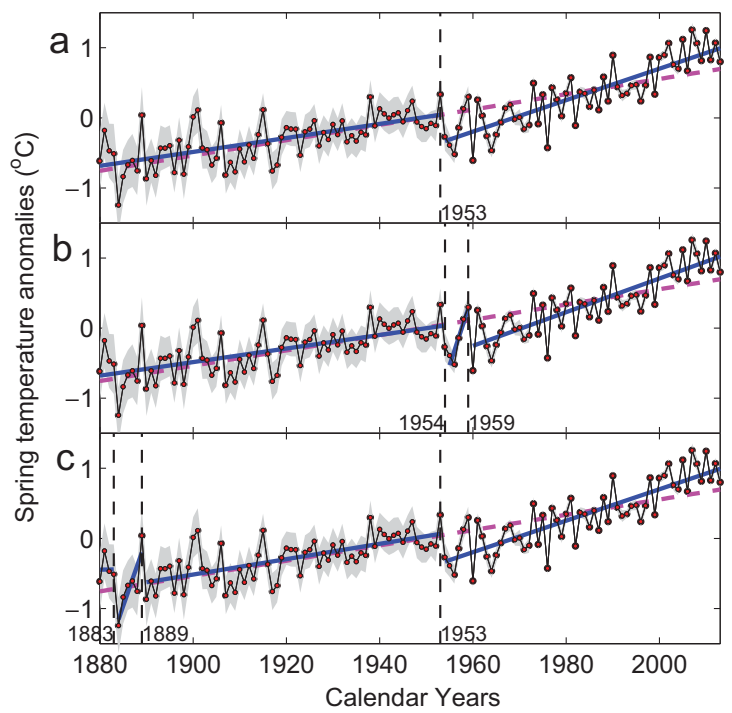

Fig. 4. Segmentation results of spring average LST anomaly time series during 1880-2013 (reference period 1901-2000). The representations is the same with 3 .

From Fig. 3a, we can see that the optimal segmentation selected by the BIC has an "increasingand-then-sharply increasing" trend over time with the change point at 1963 . This pattern is replicated except

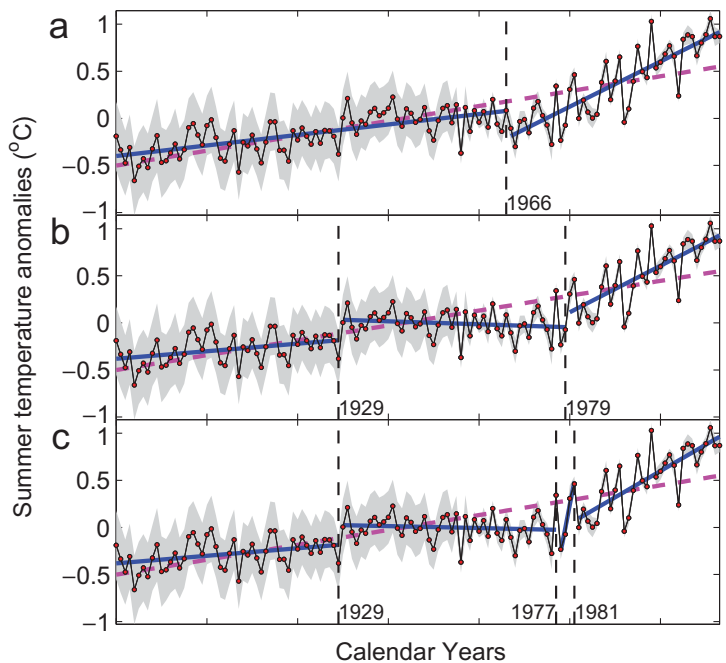

Fig. 5. Segmentation results of summer average LST anomaly time series during 1880-2013 (reference period 1901-2000). The representations is the same with Fig. 3.

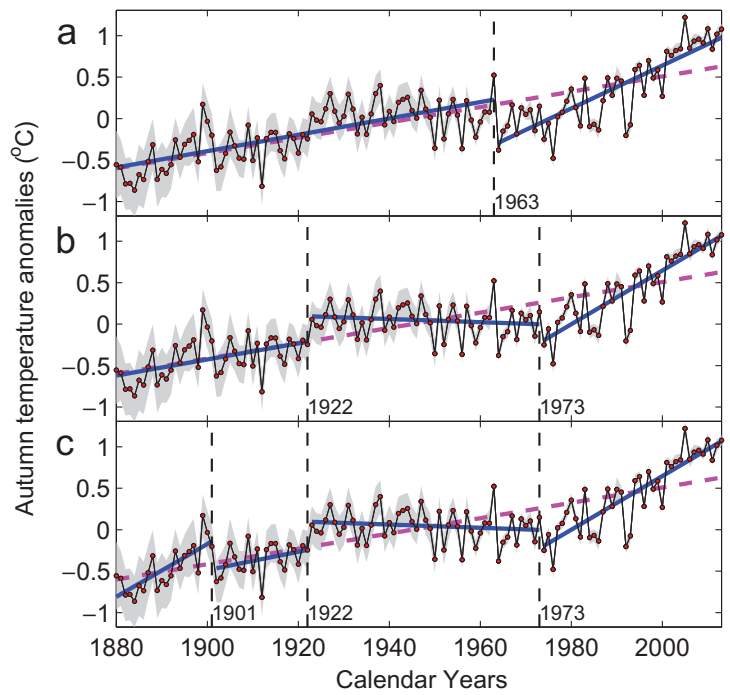

Fig. 6. Segmentation results of autumn average LST anomaly time series during 1880-2013 (reference period 1901-2000). The representations is the same with Fig. 3.

the autumn season. Segmentation results show that the warm spell started in 1880 and ended in 1963 with increasing rate $0.0093^{\circ} \mathrm{C} /$ year, while the second significant warm spell started in 1964 and ended in 2013 with positive trend $0.0244^{\circ} \mathrm{C} /$ year.

Figures 4-7 show seasonal average LST time series in the period Jan. 1880-Feb. 2014, and the partial trends up to 4th-order obtained from the DP based segmentation algorithm. It can be seen from Table 2 and Figs. 3-7 that there are some certain consisten- 


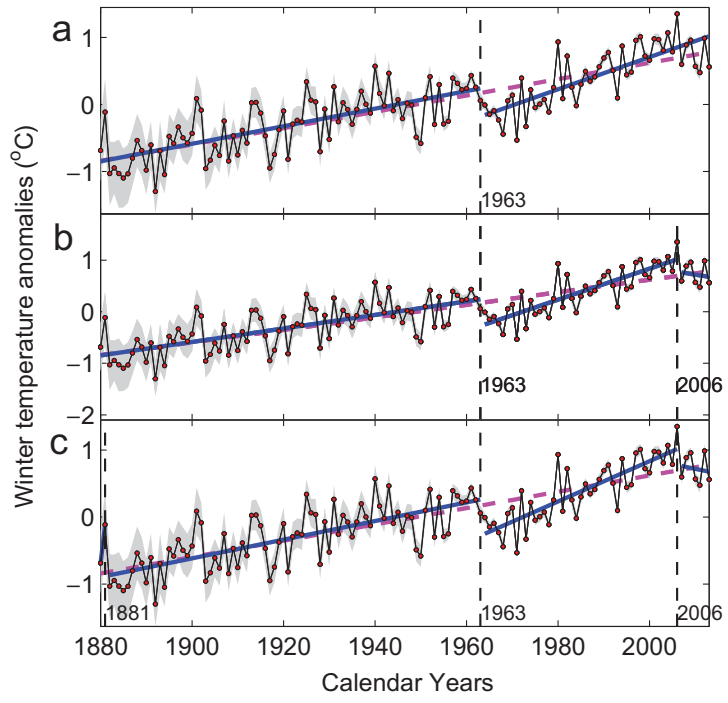

Fig. 7. Segmentation results of winter average LST anomaly time series during 1880/81-2013/14 (reference period 1901/022000/01). The representations is the same with Fig. 3.
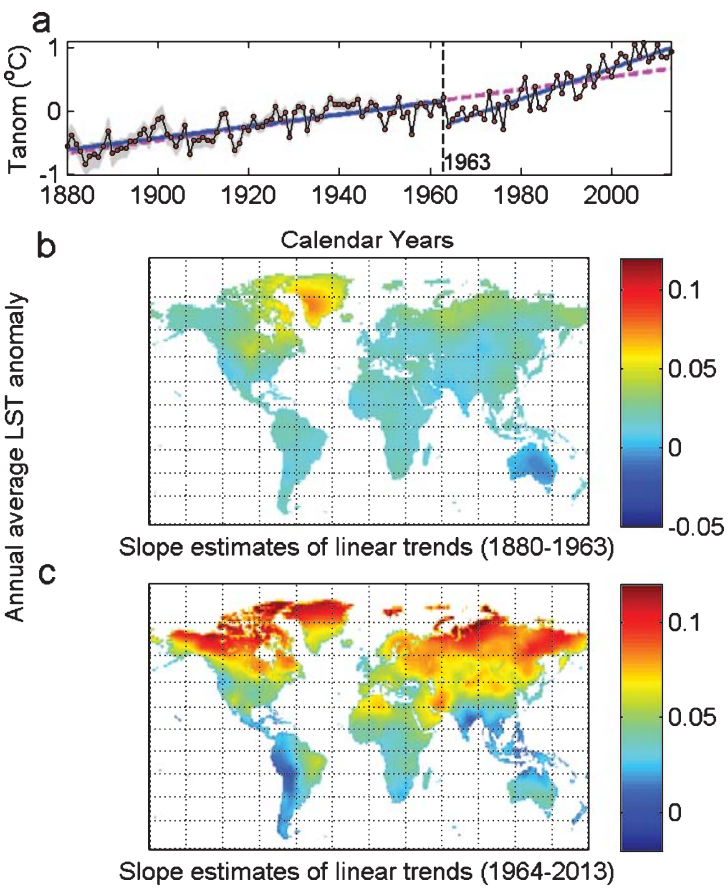

Fig. 8. Spatial distributions of linear trends (slopes) of annual LST anomalies (reference period 1901-2000) in the two periods 18801963 and 1964-2013.

cies in change points among optimal segmentations of different orders. For instance, the years 1948 and 1963 appear in annual LST anomaly time series, the year 1953 appear in spring season's, the years 1929

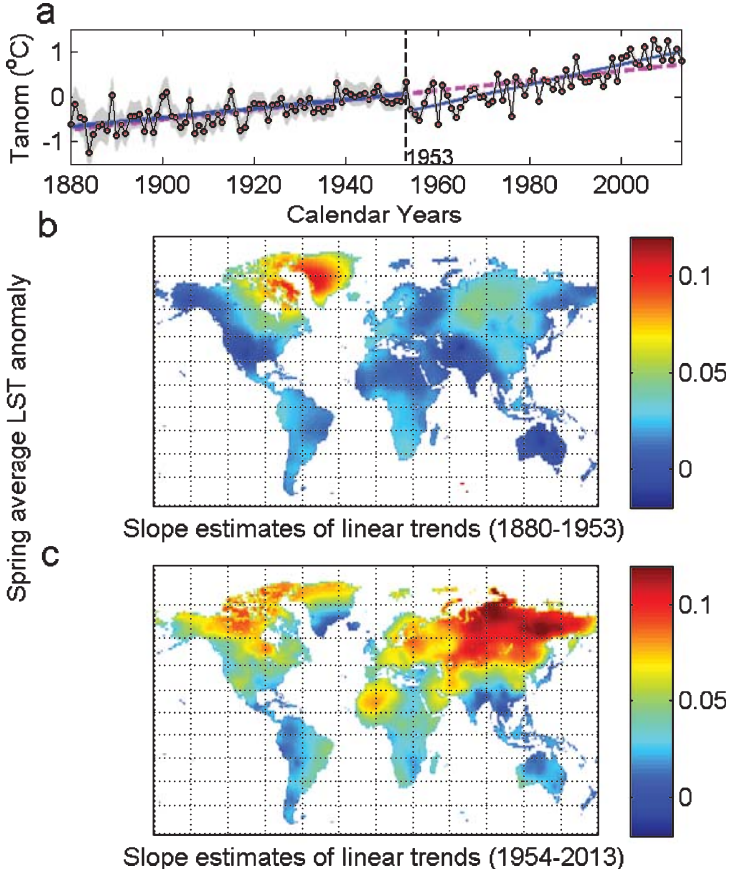

Fig. 9. Spatial distributions of linear trends (slopes) of spring LST anomalies (reference period 1901-2000) in the two periods 18801953 and 1954-2013.

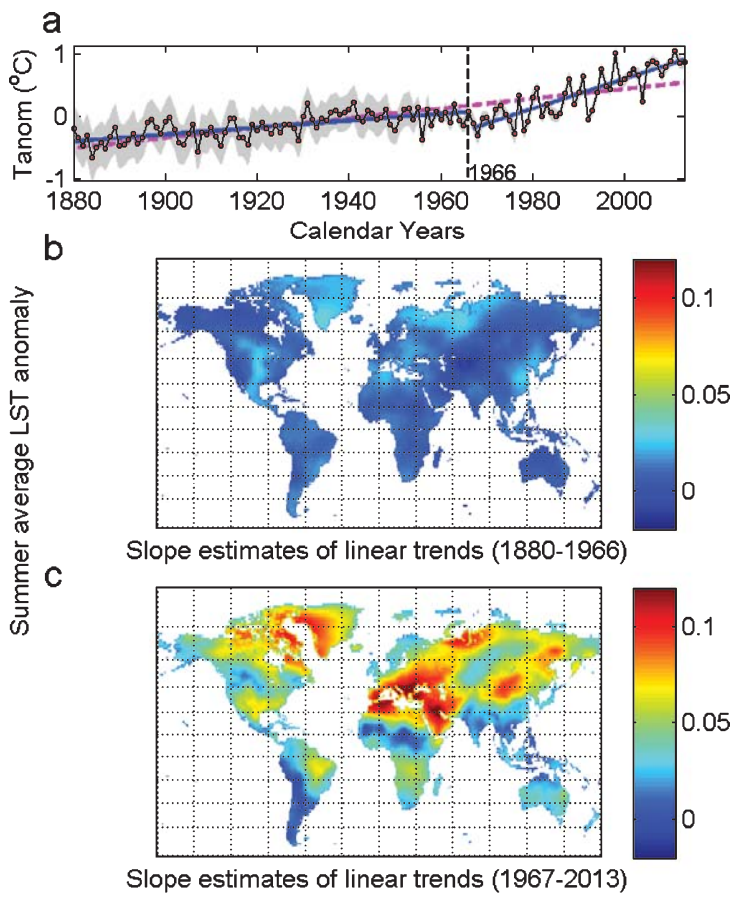

Fig. 10. Spatial distributions of linear trends (slopes) of summer LST anomalies (reference period 1901-2000) in the two periods 1880-1966 and 1967-2013. 


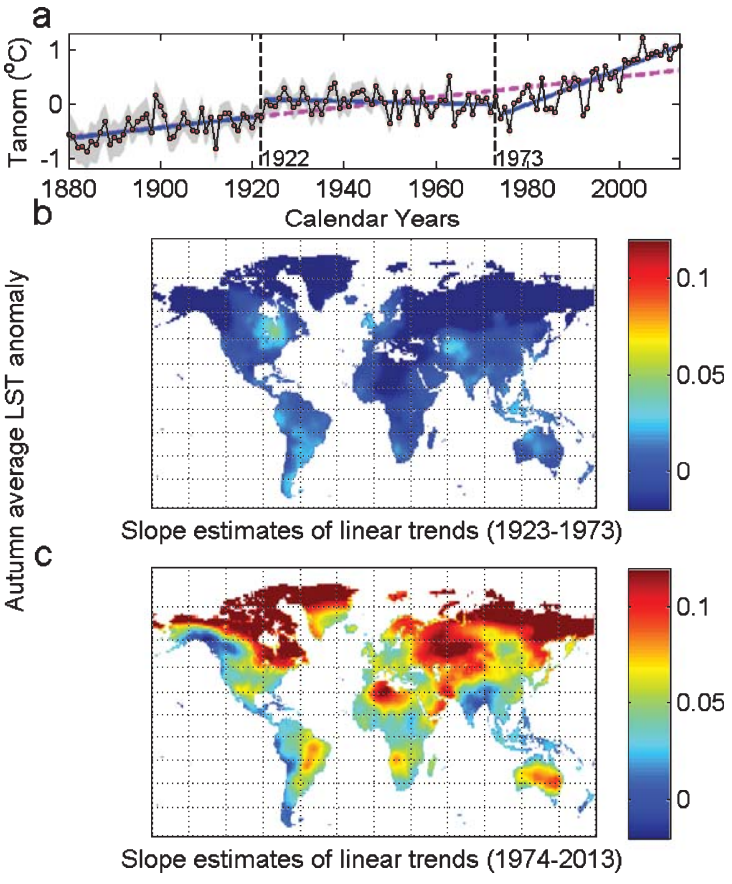

Fig. 11. Spatial distributions of linear trends (slopes) of autumn LST anomalies (reference period 1901-2000) in the two periods 1923-1973 and 1974-2013.

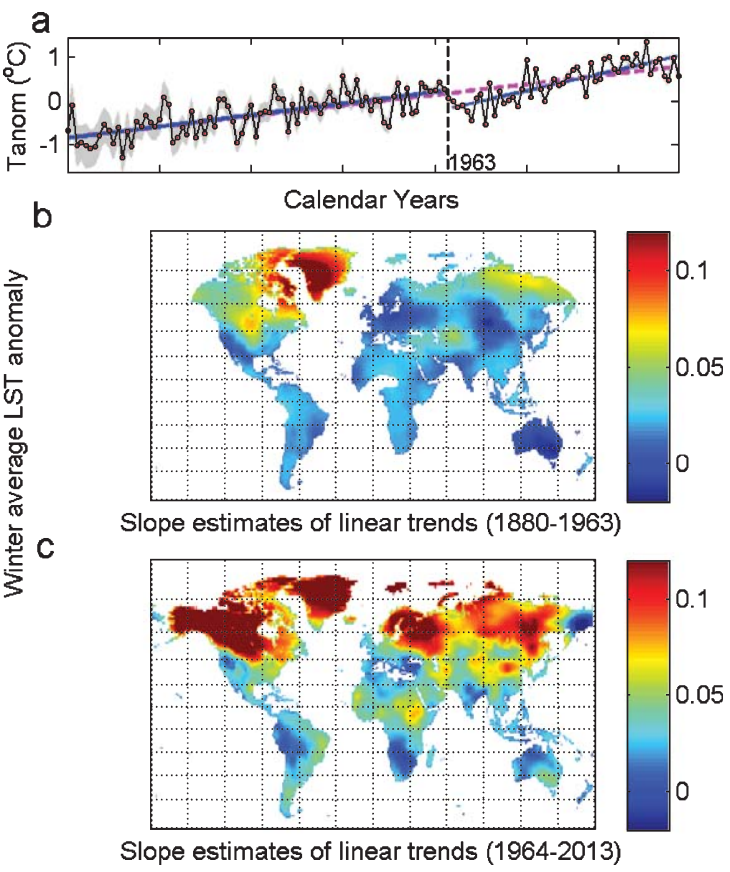

Fig. 12. Spatial distributions of linear trends (slopes) of winter LST anomalies (reference period 1901-2000) in the two periods 1880/81-1963/64 and 1964/65-2013/14. and 1977-1981 appear in summer season's, the years 1922 and 1973 appear in autumn season's, and the years 1963 and 2006 appear in winter season's. Hence some climate changes seem likely to have occurred around the years 1922, 1929, 1948, 1953-1954, 1963, 1977-1981 and 2006.

For optimal segmentation of seasonal average LST anomaly time series selected by the BIC, increasing trends are very evident. All seasons except autumn are similar to the annual average LST anomaly time seires, which have "increasing-and-then-sharply increasing" trend with different change points (1953 for spring season, 1966 for summer season, and 1963 for winter season). The spring temperature anomaly time series and partial linear trends sign (Fig. 4a) are similar to the annual series; however, the change point year is 1954 before the change point of annual series. The linear increasing rate is $0.0100^{\circ} \mathrm{C} /$ year during 1880-1954 and $0.0224^{\circ} \mathrm{C}$ /year during 1954-2013 respectively. The Summer temperature anomalies (Fig. 5a) increase with rate $0.0056^{\circ} \mathrm{C} /$ year for the period 1880-1966, followed by a remarkable warm phase $1967-2013\left(0.0240^{\circ} \mathrm{C} /\right.$ year $)$. The Autumn temperature anomalies (Fig. 6b) has a zigzag pattern "increasing-decreasing-increasing" trend over time with the change points at 1922 and 1973 . Temperatures for the 1880-1922 period are similar to other seasons and increase to persistent cold conditions until 1922 with the rate of $0.0097^{\circ} \mathrm{C} /$ year. Afterwards, a slight cold phase $\left(0.0323^{\circ} \mathrm{C} /\right.$ year $)$ is detected and this trend persists from 1923 to 1973. Then, autumn temperatures are strongly increasing $\left(0.0323^{\circ} \mathrm{C}\right)$ for the period $1974-2013$. The winter LST anomaly time series (Fig. 7a) prior to 1963/64 are increasing at the rate of $0.01311^{\circ} \mathrm{C} /$ year. After $1964 / 65$ a strong increase with rate of $0.0240^{\circ} \mathrm{C} /$ year in temperature is detected.

It is interesting to note that 1925 and 1977 are the years in which the Pacific Decadal Oscillation (PDO) changed from a cool to a warm phase, while 1947 is the year in which PDO changed from a warm to a cool phase. It is also should noted that 1922, 1949, 1954 are La Niña years, while 1929, 1963, 1982 and 2006 are El Niño years.

\subsection{Spatial trend analysis of LST anomalies from Jan. 1880 to Feb. 2014}

Based on the results of temporal variations of annual average LST anomaly time series (Fig. 8a), the spatial distributions of linear trends (slopes) of annual LST anomalies (reference period 1901-2000) during 
the period $1880-1963$ and $1964-2013$ at a $1^{\circ} \times 1^{\circ}$ (latitude $\times$ longitude) are shown in Figs. $8 \mathrm{~b}$ and $8 \mathrm{c}$. It can be noted that the trends of annual LST anomalies during these two periods are distinctly different. Figure $8 \mathrm{~b}$ shows that annual temperature in Greenland possesses a more sensitive response to climate change than other regions during the period 18801963. Figure 8c reveals that the annual warming trend has increased in almost the entire land surface during the second period 1964-2013. Northern Hemisphere exposes significant warming trend than Southern Hemisphere.

Based on the results of temporal variations of seasonal average LST anomaly time series, the spatial distributions of linear trends (slopes) of seasonal LST anomalies (reference period 1901-2000) during their respective segmentation periods at a resolution of $1^{\circ} \times 1^{\circ}$ (latitude $\times$ longitude) are shown in Figs. 9-12. From those figures, we can see the regional differences of trend for the seasonal average LST anomalies.

In spring season, the highest increasing trends of the LST anomalies are apparent in the Greenland and the surrounding area during 1880-1953 (Fig. 9b). While during 1954-2013, warming trends appear at the majority of the land, and North Asia exposes significant warming trends (Fig. 9c). The temperature warming centers are also in Northern Hemisphere, and the upward trend is relatively low in the Southern Hemisphere.

In summer season, a slightly warming trend has been found in Greenland during 1880-1966 (Fig. 10b). During 1967-2013, summer warming trend dominates most parts of land surface and significant warming trend appear along the Mediterranean coast (Fig. 10c). And West Africa is the only region that reveals a slightly decreasing trend.

In autumn season, it is clearly seen from Fig. 11b that cooling trends of LST anomaly have appeared in almost the entire region, especially in the middle-high latitudes of Northern Hemisphere during 1923-1973. While the second period 1974-2013 reveals that warming trends dominate in the middle-high latitudes of the Northern Hemisphere such as Nunavut Territory of Canada, northern Greenland, northern Asia and northern Africa (Fig. 11c).

In winter season, the highest increasing trends of the LST are apparent in the Greenland and the surrounding area during the period 1880/18811963/1964 (Fig. 12b). During the period 1964/652013/14, winter LST exposes significant warming trends in Northern Hemisphere, such as the
America-Alaska region of northern America, Yukon territory of Canada, eastern Europe, and northern Asia (Fig. 12c).

\section{Conclusions}

In order to detect climate change, this paper analyzed temporal and spatial trend changes of the annual and seasonal LST anomalies during Jan. 1880 to Feb. 2014 (reference period 1901-2000) produced by the Berkeley Earth using DP based time series segmentation algorithms.

The main conclusions are as follows:

(1) The annual and seasonal average LST anomalies have several spells, but all the temperature have upward trends, and the increasing extent of LST is the largest in winter.

(2) For the optimal segmentation results selected by BIC, only one change point is identified in annual and seasonal average LST anomaly time series except the autumn season series, and these series are seen to present overall behaviors like "increasing-and-then-sharply increasing". Two change points are identified in autumn average LST anomaly time series, and it has a zigzag pattern "increasingdecreasing-increasing" over time. Annual and seasonal average LST anomaly time series has accelerating tendency in 1960s.

(3) All the change points locate around El Niño years, La Niña years, and phase transition years of the Pacific decadal oscillation(PDO).

(4) The annual and seasonal average LST anomalies in the Northern Hemisphere possessed a more sensitive response to climate change, especially in high latitude region of the Northern Hemisphere.

(5) The DP based time series segmentation algorithm are seen to be very useful for detecting changes in the given hydro-meteorological variables. Using the method, the present work has detected important changes occurring in the annual and seasonal LST anomalies. Further studies are necessary to determine the cause of these phenomena.

\section{Acknowledgments}

This study is supported by Natural Science Foundation of China (Grant No. 41571028/51279140); 
The Applied Basic Research Project Fund of the Chinese Ministry of Transport (Grant No. 2014329225010); The General Project of Liaoning Province (Grant No. L2014214); The Fundamental Research Funds for the Central Universities of China (Grant No. 3132016116).

\section{References}

[1] A. Mackay, Climate change 2007: Impacts, adaptation and vulnerability, contribution of working group ii to the fourth assessment report of the intergovernmental panel on climate change, Journal of Environmental Quality 37 (2007), 2407.

[2] X.S. Yi, G.S. Li and Y.Y. Yin, Temperature variation and abrupt change analysis in the Three-River Headwaters Region during 1961-2010, Journal of Geographical Sciences 22 (2012), 451-469.

[3] M. Freiwan and M. Kadioğlu, Climate variability in Jordan, International Journal of Climatology 28 (2008), 69-89.

[4] H. Wang, M. Zhang, H. Zhu, X. Dang, Z. Yang and L. Yin, Hydro-climatic trends in the last 50years in the lower reach of the Shiyang River Basin, NW China, Catena 95 (2012), $33-41$.

[5] A. Burauskaite-Harju, A. Grimvall and C.V. Brömssen, A test for network-wide trends in rainfall extremes, International Journal of Climatology 32 (2012), 86-94.

[6] T.A. Buishand, G. De Martino, J.N. Spreeuw and T. Brandsma, Homogeneity of precipitation series in the Netherlands and their trends in the past century, International Journal of Climatology 33 (2013), 815-833.

[7] P. Nyeko-Ogiramoi, P. Willems and G. Ngirane-Katashaya, Trend and variability in observed hydrometeorological extremes in the Lake Victoria basin, Journal of Hydrology 489 (2013), 56-73.

[8] O.J. Olaniran, Evidence of climate change in Nigeria based on annual series of rainfall of different daily amounts, 19191985, Climatic Change 19 (1991), 319-341.

[9] H. Aksoy, A. Gedikli, N.E. Unal and A. Kehagias, Fast segmentation algorithms for long hydrometeorological time series, Hydrological Processes 22 (2008), 4600-4608.

[10] A. Gedikli, H. Aksoy and N.E. Unal, Aug-segmenter: A userfriendly tool for segmentation of long time series, Journal of Hydroinformatics 12 (2010), 318-328.

[11] T. Xiaoyong, K. Li, Z. Zeng and B. Veeravalli, A novel security-driven scheduling algorithm for precedenceconstrained tasks in heterogeneous distributed systems, IEEE Transactions on Computers 60(7) (2011), 1017-1029.

[12] Q. Shao, Z. Li and Z. Xu, Trend detection in hydrological time series by segment regression with application to Shiyang River Basin, Journal of Hydrology 24 (2010), 221-233.

[13] A.D.L. Casa and O. Nasello, Breakpoints in annual rainfall trends in Cordoba, Argentina, Atmospheric Research 95 (2010), 419-427.

[14] A.A. Ghanem, Climatology of the areal precipitation in Amman/Jordan, International Journal of Climatology 31 (2011), 1328-1333.

[15] A. Mamara, A.A. Argiriou and M. Anadranistakis, Homogenization of mean monthly temperature time series of Greece, International Journal of Climatology 33 (2013), 2649-2666.

[16] R. Mazouz, A.A. Assani, J.F. Quessy and G. Legare, Comparison of the interannual variability of spring heavy floods characteristics of tributaries of the St. Lawrence River in
Quebec (Canada), Advances in Water Resources 35 (2012), $110-120$

[17] M. Staudt, M.J. Esteban-Parra and Y. Castro-Díezm, Homogenization of long-term monthly Spanish temperature data, International Journal of Climatology 27 (2007), 1809-1823.

[18] N. Wang, X.D. Liu and J. Yin, Improved Gath-Geva clustering for fuzzy segmentation of hydrometeorological time series, Stochastic Environmental Research and Risk Assessment 26 (2012), 139-155.

[19] F. Ji, Z. Wu, J. Huang and E.P. Chassignet, Evolution of land surface air temperature trend, Nature Climate Change 4(6) (2014), 462-466.

[20] A. R. Tomé and P.M.A. Mira, Piecewise linear fitting and trend changing points of climate parameters, Geophysical Research Letters 31(2) (2004), L02207.

[21] X. Zhou, K. Li and Y. Zhou, Adaptive processing for distributed skyline queries over uncertain data, IEEE Transactions on Knowledge and Data Engineering 28(2) (2016), 371-384.

[22] W. Yang and K. Li, Performance analysis and pptimization for SpMV on GPU using probabilistic modeling, IEEE Transactions on Parallel and Distributed System 26(1) (2015), 196-205.

[23] Y. Xu, L. He, L. Zhang and K. Li, A hybrid chemical reaction optimization scheme for task scheduling on heterogeneous computing Systems, IEEE Transactions on Parallel and Distributed Systems 26(12) (2015), 3208-3222.

[24] G. Xiao, K. Li and X. Zhou, Efficient top-(k,l) range query processing for uncertain data based on multicore architectures, Distributed and Parallel Databases 33(3) (2015), 381-413.

[25] Y. Xu, L. He, L. Zhang and K. Li, A hybrid chemical reaction optimization scheme for task scheduling on heterogeneous computing Systems, IEEE Transaction on Parallel and Distributed System 26(12) (2015), 3208-3222.

[26] Y. Xu, J. Hu and K. Li, A genetic algorithm for task scheduling on heterogeneous computing systems using multiple priority queues, Information Science 270(20) (2014), 255-287.

[27] X. Zhou, K. Li and Y. Zhou, Adaptive processing for distributed skyline queries over uncertain data, IEEE Transactions on Knowledge and Data Engineering 28(2) (2016), 371-384

[28] Y. Xu, K. Li, L. He and T. Truong, A DAG scheduling scheme on heterogeneous computing systems using double molecular structure-based chemical reaction optimization, Journal of Parallel and Distributed Computing 73(9) (2013), 1306-1322.

[29] T. Truong, K. Li and Y. Xu, Chemical reaction optimization with greedy strategy for the 0-1 knapsack problem, Applied Soft Computing 13(4) (2013), 1774-1780.

[30] H. Aksoy, N. Unal, V. Alexandrov, S. Dakova and J. Yoon, Hydrometeorological analysis of northwestern Turkey with links to climate change, International Journal of Climatology 28(8) (2008), 1047-1060.

[31] C. Casty, C.C. Raible, T.F. Stocker, H. Wanner and J. Luterbacher, A european pattern climatology 1766-2000, Climate Dynamics 29(7) (2007), 791-805.

[32] A. Kehagias, A hidden Markov model segmentation procedure for hydrological and environmental time series, Stochastic Environmental Research and Risk Assessment 18 (2004), 117-130.

[33] A. Kehagias, E. Nidelkou and V. Petridis, A dynamic programming segmentation procedure for hydrological 
and environmental time series, Stochastic Environmental Research and Risk Assessment 20 (2006), 77-94.

[34] H. Aksoy, N.E. Unal and A. Gedikli, Letter to the editor, Stochastic Environmental Research and Risk Assessment 21 (2007), 447-449.

[35] A. Gedikl, H. Aksoy and N.E. Unal, Segmentation algorithm for long time series analysis, Stochastic Environmental Research and Risk Assessment 22 (2008), 291-302.
[36] P. Hubert, The segmentation procedure as a tool for discrete modeling of hydrometeorological regimes, Stochastic Environmental Research and Risk Assessment 14 (2000), 297-304.

[37] J. Yin and N. Wang, An online sequential extreme learning machine for tidal prediction based on improved Gath-Geva fuzzy segmentation, Neurocomputing 4 (2015), 243-252. 